\title{
La technique et ses conséquences
}

\section{Erhard Taverna}

Dr, membre de la rédaction

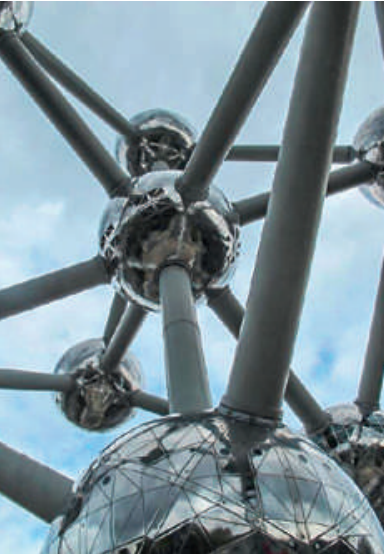

Atomium de l'année 1958: départ pour un avenir radieux.
Le dynamisme est une caractéristique de la modernité, le changement permanent est notre seule certitude. La technique permet de plus en plus de choses, avec des conséquences étendues et incontestées dans tous les domaines de la vie. Les prévisions sont de ce fait indispensables pour l'action rationnelle et la prévoyance en matière d'enjeux politiques est confiée à des professionnels de la gestion de risque.

De nombreux organismes de recherche en sociologie environnementale et technique conseillent les parlements et autorités en tentant d'évaluer les conséquences possibles des nouvelles technologies. Leur avis détermine la répartition des subventions, influence les lois et prescriptions, ainsi que le débat public lorsqu'il est question de changement climatique, de tournant énergétique, de cellules souches ou de diagnostic préimplantatoire. Aucune technique n'a que des conséquences positives ou négatives. Son évaluation passe par le recensement systématique des conséquences à attendre de son utilisation et par l'analyse des possibilités d'action en fonction de la «souhaitabilité». Idéalement, les avantages et inconvénients doivent être listés selon des critères clairs, politiquement légitimes.

Mais faire face à l'incertain en toute sécurité ne sera jamais possible-les dérives et catastrophes ne peuvent être exclues. Plus l'horizon temporel considéré est proche, plus le pronostic est fiable. L'avenir plus lointain ne peut être régi.

Amiante, CFC, fission nucléaire, génie génétique, motorisation ou numérisation, bien des exemples montrent qu'une approche purement scientifique et rationnelle, comme l'exprime la notion de gestion des risques, ne suffit pas à prévoir les conséquences à long terme. Il est tout à fait impossible d'annoncer les répercussions sociales lointaines en évaluant les options. Il faudrait pour cela une éthique d'avenir, une idée de qui nous sommes et de ce que nous voulons, une religion, une utopie, un consensus global sur les objectifs à viser. Paradoxalement, la compréhension des sciences diminue. Les croyances alternatives et superstitions augmentent en proportion de la technicisation du quotidien. Tant que les conséquences de la technique sont ressenties comme essentiellement agréables, la majorité des consommateurs s'habituera avec fatalisme à l'inévitable. Les optimistes estiment qu'on ne doit empêcher aucune évolution: le progrès s'en chargera. L'être humain est la référence ultime et n'a d'obligations qu'envers lui-même, il n'est donc pas responsable de ce qu'il trouve hors de sa sphère. Il est plus difficile de savoir qui sont les pessimistes. Sont-ce ceux qui mettent en garde contre des développements néfastes ou ceux qui jugent l'existant mauvais ou indigne au point de prendre tous les risques pour l'améliorer? Comme les promesses d'une médecine qui considère la mort comme un défaut organique, manipule la lignée germinale ou permet des contrôles de comportements sociaux.

Conscient du vide éthique, le philosophe Hans Jonas (1903-1993) a écrit Le principe Responsabilité - Une éthique pour la civilisation technologique [1]. Faute de meilleures représentations, il recommandait de don-

Faute de meilleures représentations, Jonas recommandait de donner la priorité au pronostic pessimiste.

ner la priorité au pronostic pessimiste. Pour les risques incertains avec des conséquences potentielles de grande ampleur, il préconisait une «heuristique de la peur». Si le bien à sauver est discernable dans le mal, si le bonheur non illusoire et dépassé peut être décelé dans le malheur, alors la peur est le premier devoir d'une éthique de la responsabilité historique. Seul le respect de ce que l'Homme est et a été protège des égarements du pouvoir. La modestie et le renoncement n'ont jamais été populaires. Sa mise en garde contre le complexe de supériorité de l'Homme ne figure pas sur la check-list des instituts actuels d'évaluation des technologies. Elève de Heidegger et Bultmann, Hans Jonas a écrit un ouvrage de référence sur l'histoire du gnosticisme. Il a vécu l'exil, la guerre et la terreur de la shoah. Son témoignage et ses immenses connaissances historiques modèlent son analyse philosophique de la «responsabilité», notamment des sciences médicales. Son raisonnement ouvre une dimension qui va bien au-delà de la simple gestion de risque. «Le respect seul, dans la mesure où il nous dévoile quelque chose de ssacrés, c'est-à-dire quelque chose qui en aucun cas ne doit être atteint (et cela peut être entrevu même en l'absence de religion positive), nous protégera contre la tentation de violer le présent au bénéfice de l'avenir, de vouloir acheter celui-là au détriment de celui-ci», selon Jonas.

1 Jonas H. Le principe Responsabilité. Paris: Les éditions du Cerf 1990 . 